\title{
Negative Hydrogen Ion Source Research and Beam Parameters for Accelerators
}

\author{
Timofey V. Zolkin \\ Supervisors: Douglas P. Moehs and Charles W. Schmidt \\ Fermilab, P.O. Box 500, Batavia, IL 60510, U.S.A.
}

\section{Abstract}

$\mathrm{H}^{-}$beams are useful for multi-turn charge-exchange stripping injection into circular accelerators. Studies on a modified ion source for this purpose are presented. This paper includes some theory about a $\mathrm{H}^{-}$magnetron discharge, ion-electron emission, emittance and problems linked with emittance measurement and calculations.

Investigated parameters of the emittance probe for optimal performance give a screen voltage of $150 \mathrm{~V}$ and a probe step of about 5 mil. Normalized 90\% emittance obtained for this $\mathrm{H}^{-}$source is $0.22 \pi \mathrm{mm}-\mathrm{mr}$, for an extraction voltage of $18 \mathrm{kV}$ at a beam energy of $30 \mathrm{keV}$ and a beam current of $11 \mathrm{~mA}$.

\section{Key Words}

Accelerator, beam, plasma, ion source, emittance, ionelectron emission, emittance measurement, negative hydrogen.

\section{INTRODUCTION}

At Fermi National Accelerator Laboratory (USA) $\mathrm{H}^{-}$ sources are based on a magnetron discharge. For 30 years it has been the primary source for $\mathrm{H}^{-}$beams. The Linac uses a source in which the beam is magnetically bent 90 degrees. This magnetic field focuses the beam in one direction and unfortunately defocuses in the other. Now there is an experiment to obtain a beam that is extracted without bending for injection into an RFQ. Initially the source used a flat cathode (like an oval prism), later a half-cylinder groove gave better performance, and now a half-spherical indentation is being used (see Fig. 1). This paper will describe emittance data for the source with a spherical groove.

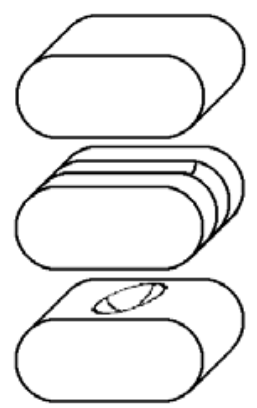

Figure 1: Types of cathodes for the magnetron source.

The second issue we will describe is collecting the data and calculating emittance from raw data.
THEORY

\section{Ion source}

One process by which $\mathrm{H}^{-}$ions are produced will be explained using Fig. 2. A proton or other ion $\left(\mathrm{Cs}, \mathrm{Mo}, \mathrm{O}_{2}\right.$, $\mathrm{N}_{2}$, etc.) from the plasma strikes the cathode removing a desorbed hydrogen atom or as a proton reflects from the cathode surface. As the particles leave the surface some capture an extra electron and leave as a $\mathrm{H}^{-}$. The $\mathrm{H}^{-}$ions then pass through the plasma to be extracted. The number of $\mathrm{H}^{-}$ions which are then extracted from the source depend strongly on the distance through the plasma and destruction reactions (Fig. 3 and Table 1).

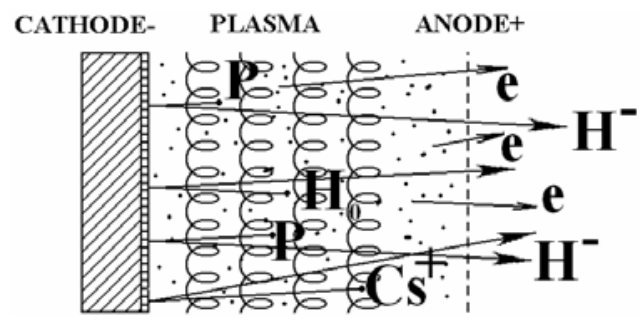

Figure 2: Plasma processes.[6]

Fig. 3 shows the dominate types of processes while table 1 gives the processes, with maximum cross section and corresponding reaction rates.

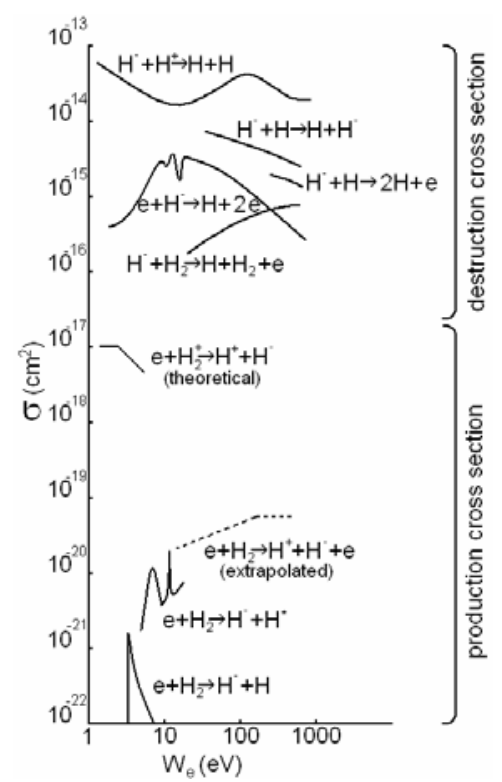

Figure 3: Negative hydrogen production and destruction cross sections as a function of energy.[4] 


\begin{tabular}{|c|c|c|c|c|}
\hline Process & Reaction & $\sigma \max (\mathrm{cm} 2)$ & $\begin{array}{l}\text { Energy for } \\
\text { omax }(\mathrm{eV})\end{array}$ & $\begin{array}{l}\text { Reaction (at kT) } \\
\langle\sigma\rangle(\mathrm{cm} 3 / \mathrm{sec})\end{array}$ \\
\hline \multicolumn{5}{|c|}{ Production } \\
\hline Di ssociative attachment & $\begin{array}{l}\mathrm{e}+\mathrm{H}_{2} \rightarrow \mathrm{H}^{-}+\mathrm{H} \\
\mathrm{e}+\mathrm{H}_{2} \rightarrow \mathrm{H}^{-}+\mathrm{H}^{*}\end{array}$ & $\begin{array}{l}1.6 \times 10^{-21} \\
2.1 \times 10^{-20}\end{array}$ & $\begin{array}{l}3.7 \\
14\end{array}$ & $5 \times 10^{-13}\left(4 e^{V}\right)$ \\
\hline Polar dissociation & $\mathrm{e}+\mathrm{H}_{2} \rightarrow \mathrm{H}^{-}+\mathrm{H}^{+}+\mathrm{e}$ & $\begin{array}{l}1.7 \times 10^{-20} \\
\text { and rising }\end{array}$ & 38 & $3 \times 10^{-12}(15 \mathrm{eV})$ \\
\hline Dissociative recombination & $\mathrm{e}+\mathrm{H}_{2}^{+} \rightarrow \mathrm{H}^{-}+\mathrm{H}^{+}$ & $10^{-17}$ & 3 & $3 \times 10^{-10}(3 \mathrm{eV})$ \\
\hline Charge exchange & $\mathrm{H}+\mathrm{H}^{-} \rightarrow \mathrm{H}^{-}+\mathrm{H}$ & $8 \times 10^{-15}$ & 40 & \\
\hline Radiative capture & $\mathrm{e}+\mathrm{H} \rightarrow \mathrm{H}^{-}+\mathrm{h} \nu$ & $2.6 \times 10^{-22}$ & 0.7 & $10^{-14}(1 \mathrm{eV})$ \\
\hline \multicolumn{5}{|c|}{ Destruction } \\
\hline Collisional detachment & $\begin{array}{l}\mathrm{e}+\mathrm{H}^{-} \rightarrow \mathrm{H}+2 \mathrm{e} \\
\mathrm{H}^{-}+\mathrm{H} \rightarrow 2 \mathrm{H}+\mathrm{e}\end{array}$ & $\begin{array}{l}4 \times 10^{-15} \\
1.6 \times 10^{-15}\end{array}$ & $\begin{array}{l}15 \\
500\end{array}$ & $7 \times 10^{-7}(15 \mathrm{eV})$ \\
\hline Associative detachment & $\mathrm{H}^{-}+\mathrm{H} \rightarrow \mathrm{H}_{2}+\mathrm{e}$ & & & $10^{-9}(\sim 1 \mathrm{eV})$ \\
\hline Collisional detadhment & $\mathrm{H}^{-}+\mathrm{H}_{2} \rightarrow \mathrm{H}+\mathrm{H}_{2}+\mathrm{e}$ & $10^{-15}$ & $10^{4}$ & \\
\hline Charge transfer & $\mathrm{H}^{-}+\mathrm{H}^{+} \rightarrow 2 \mathrm{H}$ & $2.5 \times 10^{-13}$ & $0.15(\mathrm{~cm})$ & $5 \times 10^{-7}(<1 \mathrm{keV})$ \\
\hline & $\mathrm{H}^{-}+\mathrm{H}_{2}^{+} \rightarrow \mathrm{H}+\mathrm{H}_{2}$ & & & $\sim 10^{-7}$ \\
\hline Di ssociative attachment & $\mathrm{H}^{-}+\mathrm{H}_{2} \mathrm{O} \rightarrow \mathrm{OH}^{-}+\mathrm{H}_{2}$ & & 2 & $3 \times 10^{-8}(2 \mathrm{eV})$ \\
\hline
\end{tabular}

Table 1: Maximum values of cross section and corresponding energies for elementary processes leading to the production or destruction of a negative hydrogen ion. The corresponding energy is indicated in parentheses.[4]

The reaction rates are defined as the number of collisions of a given kind per unit density of interacting particles and per unit time. (Note: the reaction rates for the destruction processes are typically several orders larger than the production rates and significantly affect the design of a $\mathrm{H}^{-}$source). Accordingly one process can only be compared to another, by integrating over the velocity distribution function $\mathrm{f}(\mathrm{v})$ of the fast particle

$$
\langle\sigma v\rangle=\int_{0}^{\infty} \sigma(\mathrm{v}) \mathrm{vf}(\mathrm{v}) \mathrm{dv} \text {. }
$$

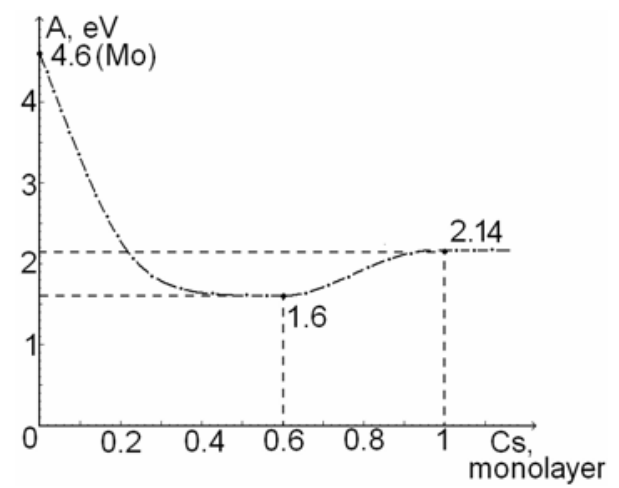

Figure 4: Work function of Mo as a function of a fractional Cs monolayer on a Mo surface.

One of the most important process is when the proton hits the cathode surface, and must collect two electrons. For clean Mo the work function is very large, $4.6 \mathrm{eV}$, and only one electron is likely to be transferred. To significantly increase the captured electrons, Cs vapor is injected onto the Mo surface to reduce the work function. Fig. 4 shows the change in work function as Cs is added. The lowest value is with $\mathrm{Cs}$ at 0.6 of a monolayer, where the work function has a minimum (about $1.6 \mathrm{eV}$ ). Here the $\mathrm{H}^{-}$production is a maximum.

Details of the FNAL H${ }^{-}$source are shown in figures 5 and 6 . Reducing the distance between the cathode and anode causes more $\mathrm{H}^{-}$particles to be preserved until they are extracted. However, if the distance is less than the Larmor radius of the electrons, they will be lost on the boundaries. This will cause a low density plasma and low $\mathrm{H}^{-}$production.

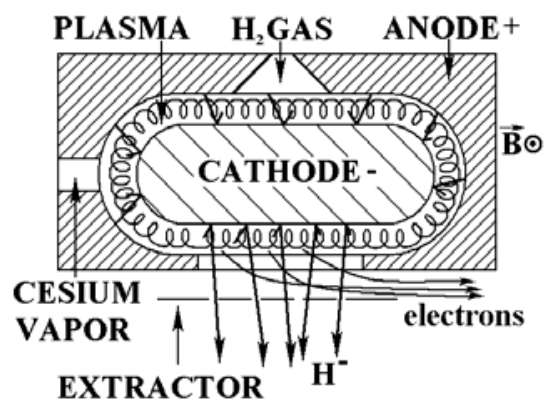

Figure 5 : Magnétron type source.[6]

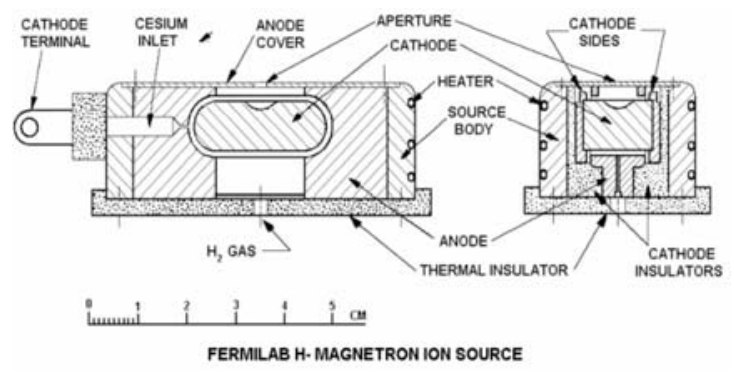

Figure 6 : FERMILAB H magnétron source.[6] 


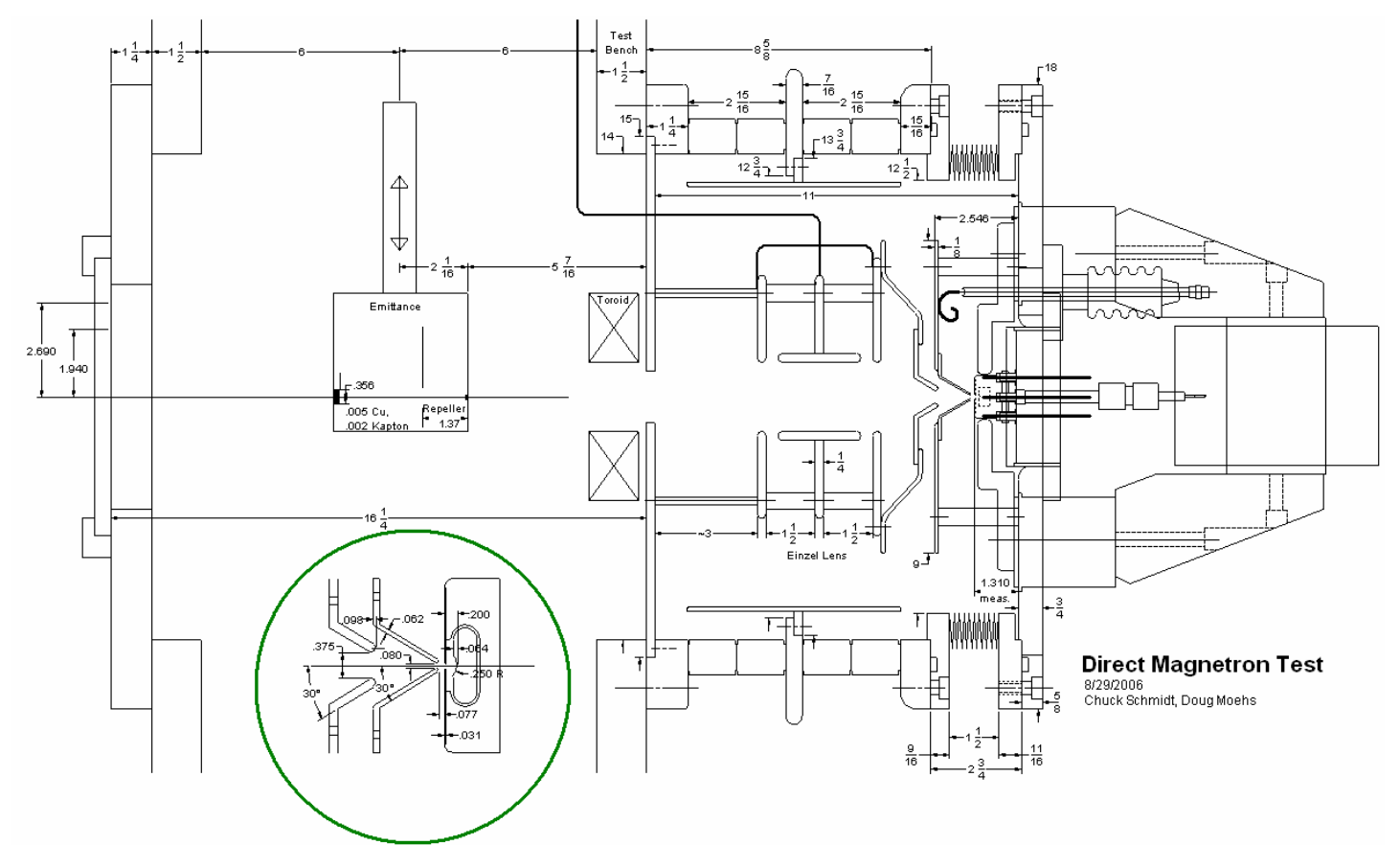

Figure 7: Direct extraction magnetron test.[5]

\section{Setup}

The beam is extracted with $18 \mathrm{kV}$. After extraction the beam accelerates to $30 \mathrm{keV}$ by the voltage gradient between the source and final accelerating electrode, (see fig. 7). Following extraction and acceleration an einzel lens is used for focusing. The lens voltage is usually about $21 \mathrm{kV}$, but can change depending on parameters of specific experiment. After the lens a toroid measures the beam current. Following the toroid are the two emittance probes for vertical and horizontal measurements.

\section{Emittance probe}

An emittance probe consist of an entrance slit, screen electrode and target composed of 50 electrode strips (see Fig. 8 and 9).

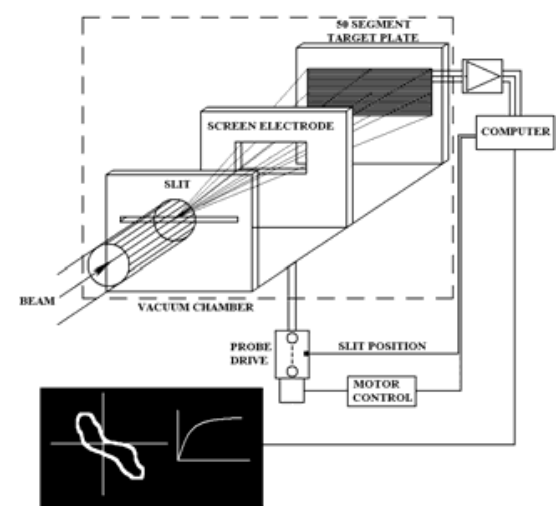

Figure 8: Emittance probe design.

The probe is moved by a stepping motor with the position defined and measured by a computer. When a particle enters the probe slit and passes the screen electrode it hits one of the target electrodes which defines the particle angle. The screen electrode is used to control the electrons emitted from the target electrodes, when an $\mathrm{H}^{-}$ion hits an electrode, electrons are emitted and the data can be affected (see Fig. 10). This process is described in the experimental part.

Two probes scan the beam in the vertical and horizontal dimensions.

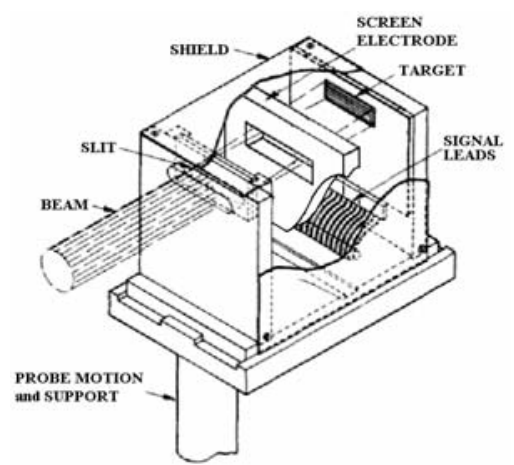

Figure 9: Detail of emittance probe.[6]
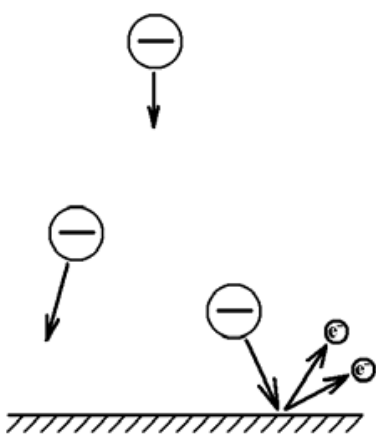

Figure 10: Ion-electron emission. 


\section{Beam Emittance}

The basic equation of emittance $\left(A_{6}\right)$ is

$A_{6}=\iiint p_{x} p_{y} p_{z} d x d y d z=$ const,

which is derived from Liouvile's law if only conservative forces are acting. Assuming all forces of motion in each direction are decoupled then Liouvile's law applies separately to each plane. The definition of 2-dimension emittance becomes:

$$
\frac{\mathrm{A}_{2}}{\pi \mathrm{mc}}=\frac{1}{\pi \mathrm{mc}} \int \mathrm{p}_{\mathrm{x}} \mathrm{dx}=\frac{1}{\pi} \int \beta_{\mathrm{x}} \gamma_{\mathrm{x}} \mathrm{dx}=\varepsilon_{\mathrm{n}}
$$

where $\varepsilon_{\mathrm{n}}$ is the normalized emittance, and

$\beta_{\mathrm{x}}=\frac{\mathrm{v}_{\mathrm{x}}}{\mathrm{c}}$ and $\gamma_{\mathrm{x}}=\frac{1}{\sqrt{1-\beta_{\mathrm{x}}{ }^{2}}}$.

Often emittance is given in terms of "laboratory" emittance, which is:

$\varepsilon=\frac{\varepsilon_{\mathrm{n}}}{\beta_{\mathrm{x}} \gamma_{\mathrm{x}}}=\frac{\int \beta_{\mathrm{x}} \gamma_{\mathrm{x}} \mathrm{dx}}{\pi \beta_{\mathrm{x}} \gamma_{\mathrm{x}}}=\frac{1}{\pi} \int \mathrm{x}^{\prime} \mathrm{dx}$.

The ideal emittance is equal to zero, but this is unrealistic for real ion beams (see Fig. 11).

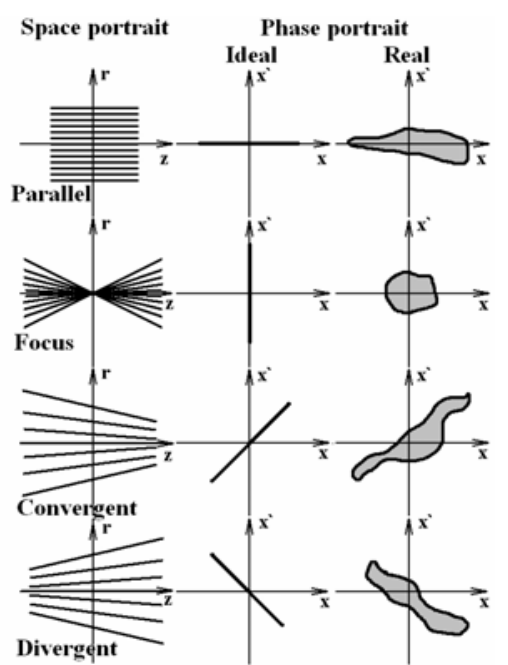

Figure 11: Ideal and real emittance for different cases.

Real emittance is always larger than zero, implying that particles have varied motion in real beams. In theory it is not difficult to calculate emittance. However, when working with real data the situation changes very dramatically. First we shall show what is usually done to calculate emittance and then discuss real situations later.

Emittance is represented by properties of an ellipse in phase space (Fig. 12):

1. Analytical parameters:

$$
\begin{aligned}
& x^{\prime}= \pm \frac{b}{a} \sqrt{a^{2}-x^{2}}+c x \Rightarrow \varepsilon=a \cdot b \\
& a=x_{\text {max }} ; b=x_{0}^{\prime}
\end{aligned}
$$

(for a centered ellipse)
2. Twiss parameters:

$$
\begin{aligned}
& \widetilde{\gamma} x^{2}+2 \widetilde{\alpha} x^{\prime}+\widetilde{\beta} x^{\prime 2}=\widetilde{\varepsilon}, \\
& \text { with } \widetilde{\beta} \widetilde{\gamma}-\widetilde{\alpha}^{2}=1 ; \widetilde{\beta}, \widetilde{\gamma}>0 .
\end{aligned}
$$

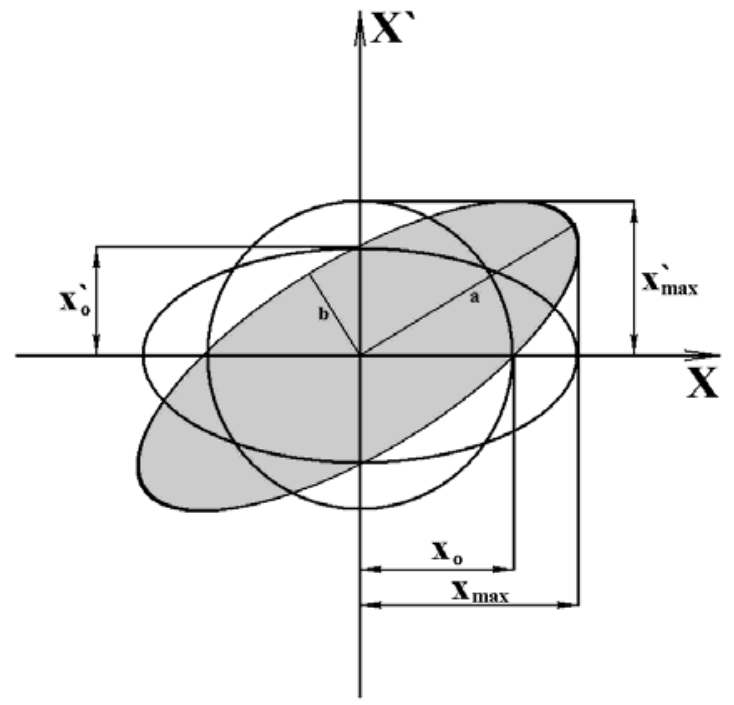

Figure 12: Representation of emittance as an ellipse.

Ellipses with identical parameters $\alpha, \beta$ have the same shape, even for different $\varepsilon$ values. Some authors use the area of the ellipse figure, rather than area/ $\pi$ as the emittance. As a compromise for distinguishing true versus area emittance, the factor $\pi$ is put into the dimension (i.e., $\varepsilon=1.23 \pi \mathrm{mm}$-mrad).

Often it is necessary to use fractional emittance because it is possible to analysis only part of the beam. For fractional emittance one measures the $100 \%$ emittance in $\mathrm{x} \mid \mathrm{x}^{`}$ and $\mathrm{y} \mid \mathrm{y}$, then removes particles below a desired current threshold, and finally computes the emittance based on the remaining distributions (see Fig. 13). It is important that this procedure is treated in four dimensions! (x|x` and $\left.y \mid y^{\prime}\right)$.

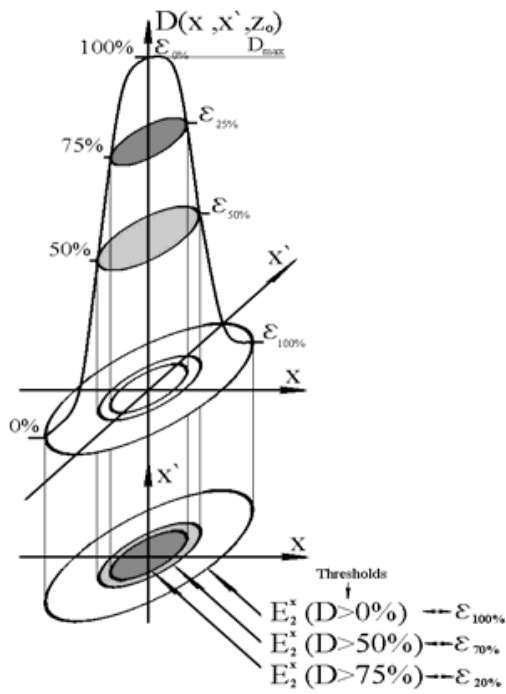

Figure 13: The beam fraction.[1] 
To obtain numerical values of "equivalent ellipses", parameters from discrete particle distributions, the RMS emittance is useful.

$$
\begin{aligned}
& \varepsilon_{\text {RMS }}=\sqrt{\left\langle\mathrm{x}^{\prime 2}\right\rangle\left\langle\mathrm{x}^{2}\right\rangle-\left\langle\mathrm{xx}^{\prime}\right\rangle^{2}}, \\
& \text { with } \\
& \left\langle\mathrm{x}^{2}\right\rangle=\frac{\sum_{\text {all }} \mathrm{x}^{2} \mathrm{c}\left(\mathrm{x}, \mathrm{x}^{\prime}\right)}{\sum_{\text {all }} \mathrm{c}\left(\mathrm{x}, \mathrm{x}^{\prime}\right)},\left\langle\mathrm{x}^{\prime 2}\right\rangle=\frac{\sum_{\text {all }} \mathrm{x}^{\prime 2} \mathrm{c}\left(\mathrm{x}, \mathrm{x}^{\prime}\right)}{\sum_{\text {all }} \mathrm{c}\left(\mathrm{x}, \mathrm{x}^{\prime}\right)}, \\
& \left\langle\mathrm{Xx}^{\prime}\right\rangle=\frac{\sum_{\text {all }} \mathrm{x} \mathrm{x}^{\prime} \mathrm{c}\left(\mathrm{x}, \mathrm{x}^{\prime}\right)}{\sum_{\text {all }} \mathrm{c}\left(\mathrm{x}, \mathrm{x}^{\prime}\right)}
\end{aligned}
$$

Where $\mathrm{c}\left(\mathrm{x}, \mathrm{x}^{\prime}\right)$ is the beam current at a given position and velocity in the beam cross-section, $\mathrm{x}, \mathrm{x}^{\prime}$, with the first moment terms evaluate to be zero, namely:

$$
\langle\mathrm{x}\rangle=\frac{\sum_{\text {all }} \mathrm{xc}\left(\mathrm{x}, \mathrm{x}^{\prime}\right)}{\sum_{\text {all }} \mathrm{c}\left(\mathrm{x}, \mathrm{x}^{\prime}\right)}=0,\left\langle\mathrm{x}^{\prime}\right\rangle=\frac{\sum_{\text {all }} \mathrm{x}^{\prime} \mathrm{c}\left(\mathrm{x}, \mathrm{x}^{\prime}\right)}{\sum_{\text {all }} \mathrm{c}\left(\mathrm{x}, \mathrm{x}^{\prime}\right)}=0
$$

This renormalization is equivalent to maximizing the beam transmission corresponding to steering of the beam and minimizing the emittance. The orientation and aspect ratio of the RMS-emittance ellipse is described by the Twiss parameters, namely

$$
\widetilde{\alpha}=-\frac{\left\langle\mathrm{xx}^{\prime}\right\rangle}{\varepsilon}, \widetilde{\beta}=-\frac{\left\langle\mathrm{x}^{2}\right\rangle}{\varepsilon}, \widetilde{\gamma}=-\frac{\left\langle\mathrm{x}^{\prime 2}\right\rangle}{\varepsilon} \text {. }
$$

The RMS emittance is usually used when the beam distribution is not simple. In these cases it is a method for

\begin{tabular}{|c|c|c|c|}
\hline Beam size & Emittance & Fraction & \\
\hline$\pm\left\langle x^{2}\right\rangle^{1 / 2}$ & $\pi \sigma_{\mathrm{h}}^{2}$ & $39.3 \%$ & RMS \\
\hline $\pm 2\left\langle x^{2}\right\rangle^{1 / 2}$ & $4 \pi \sigma_{\mathrm{h}}^{2}$ & $86.5 \%$ & CERN \\
\hline $\begin{array}{l} \pm \text { FWHM } \\
\left( \pm 2.355\left\langle x^{2}\right\rangle^{1 / 2}\right)\end{array}$ & $5.54 \pi \sigma_{\mathrm{h}}^{2}$ & $93.75 \%$ & \\
\hline $\begin{array}{l} \pm \sqrt{6}\left\langle x^{2}\right\rangle^{1 / 2} \\
\left( \pm 2.449\left\langle x^{2}\right\rangle^{1 / 2}\right)\end{array}$ & $6 \pi \sigma_{\mathrm{h}}^{2}$ & $95.0 \%$ & FNAL \\
\hline $\pm 2.145\left\langle\mathrm{x}^{2}\right\rangle^{1 / 2}$ & $4.60 \pi \sigma_{\mathrm{h}}^{2}$ & $90 \%$ & $\begin{array}{l}\text { FNAL } \\
\text { Linac }\end{array}$ \\
\hline
\end{tabular}
estimating emittance.

In addition to this part of the theory we must describe different definitions of emittance. Table 2 gives the beam fraction used for some conditions and at some laboratories, CERN and FNAL for example.

Table 2: Emittance fraction used at different places.[3]

\section{EXPERIMENTAL PART}

As explained earlier, part of this paper is to understand the emittance measurement process which happens when we collect data. The second purpose is to understand the beam emittance.

Initially there was considerable negative data in the emittance plot believed due to electrons scattered from the target electrodes (Fig. 14) and one could observe significant changes in the low level data with different screen voltages. It became necessary to show what happens for positive and negative screen voltages to explain this process.

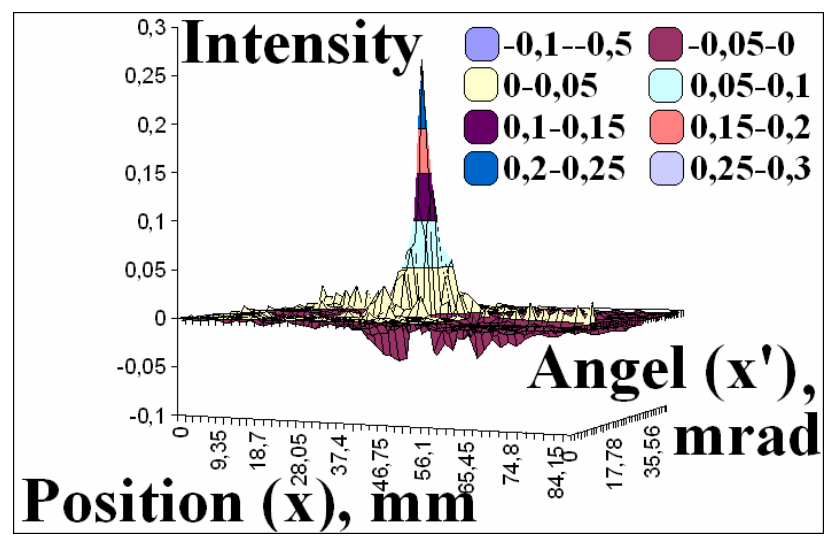

Figure 14: Illustration of typical collected data.

Figures 15 to 17 display raw data with a minus screen voltage in a 3-D plot and sliced plots with angle on the abscissa and intensity on the ordinate. There is a negative hole around the $\mathrm{H}^{-}$and other peaks. Figure 18 shows what happens. At the center of the beam many $\mathrm{H}^{-}$particles hit the target electrodes and knock out several electrons per $\mathrm{H}^{-}$ion. With a minus screen voltage the electric field returns the electrons to various target electrodes. In areas were the beam is low the electrons leave a negative signal (the electrons return to the wires like a splash in water). In this manner the real data is greatly distorted.

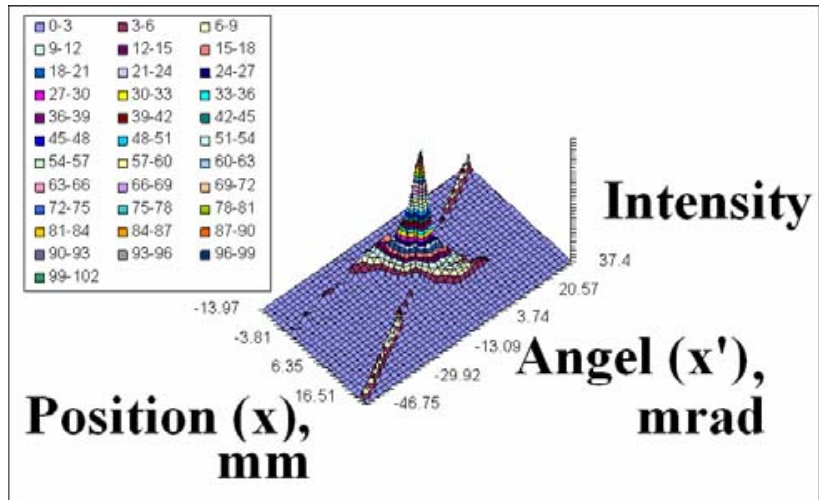

Figure 15: 3-D plot of $\mathrm{H}^{-}$beam $\mathrm{X}$ emittance data with negative screen voltage $(-600 \mathrm{~V})$. 


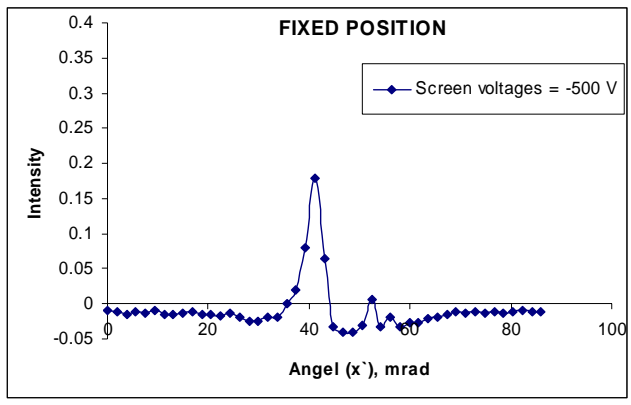

Figure 16: Slice of 3-D plot of $\mathrm{H}^{-}$beam $\mathrm{X}$ emittance data with negative screen voltage $(-500 \mathrm{~V})$.

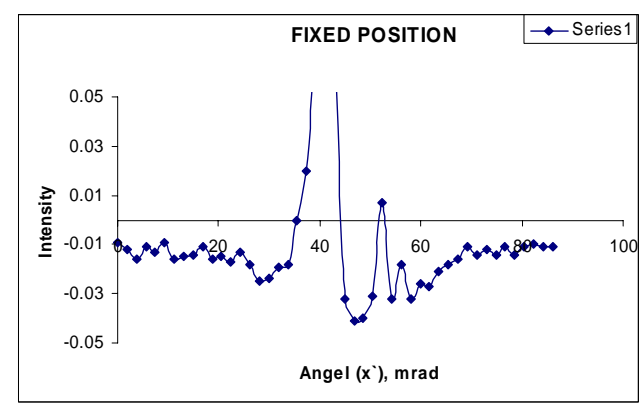

Figure 17: Slice of 3-D plot of $\mathrm{H}^{-}$beam $\mathrm{X}$ emittance data with negative screen voltage $(-500 \mathrm{~V})$. (Intensity scale increased)

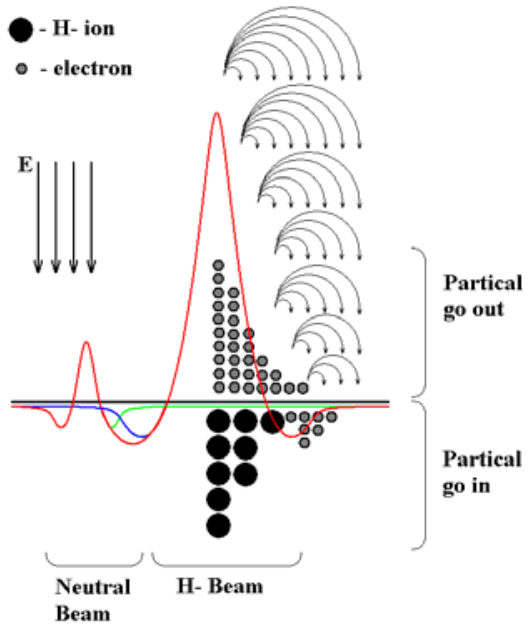

Figure 18: Model of process with negative screen voltages.

The next four figures represent the same test but for a positive screen voltage. There is no negative area in the plots with positive screen voltage at this beam current, see figures 19 to 21 . But in the sliced data there are noticeably wide shoulders around the beam area. In Fig. 22 , the model is similar to the negative screen voltage but with a strong positive screen voltage the electric field is reversed and this extracts the electrons. Here the signals are slightly stronger but large shoulders seem to appear due to excess electrons being extracted by the high electric field. Therefore it is necessary to find a compromise between these two processes.

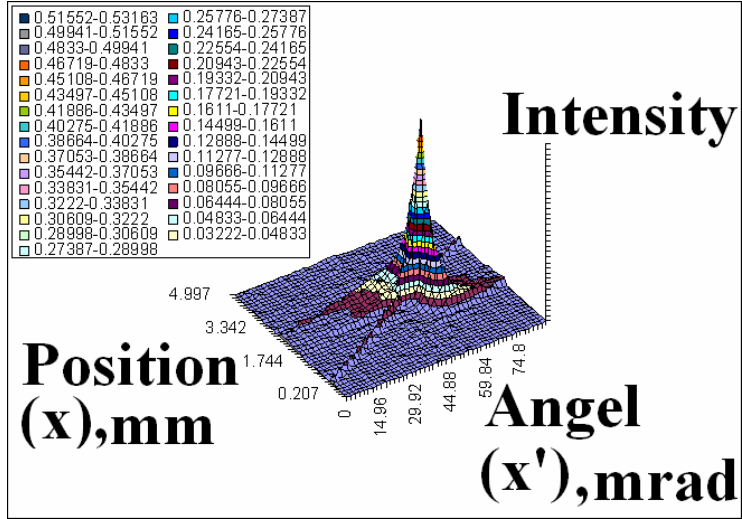

Figure 19: 3-D plot of $\mathrm{H}^{-}$beam $\mathrm{X}$ emittance data with positive screen voltage $(1000 \mathrm{~V})$.

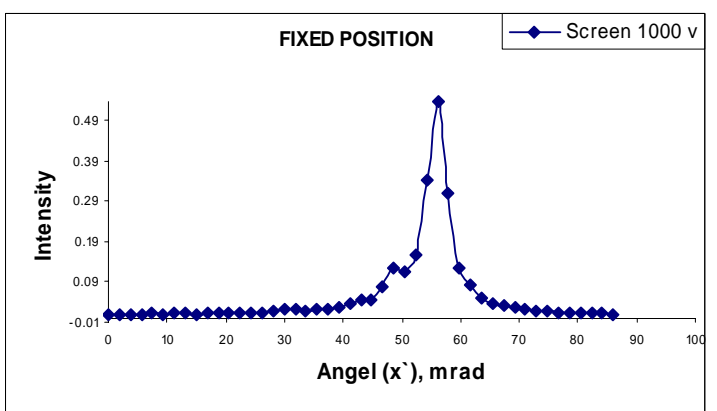

Figure 20: Slice of 3-D plot of $\mathrm{H}^{-}$beam $\mathrm{X}$ emittance data with positive screen voltage $(1000 \mathrm{~V})$.

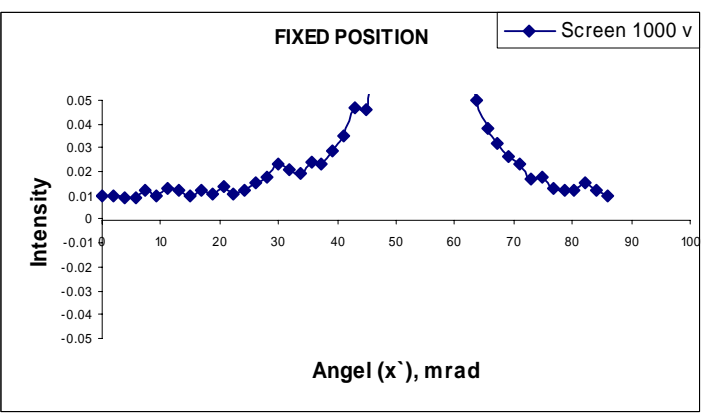

Figure 21: Slice of 3-D plot of $\mathrm{H}^{-}$beam $\mathrm{X}$ emittance data with positive screen voltage $(1000 \mathrm{~V})$. ). (Intensity scale increased)

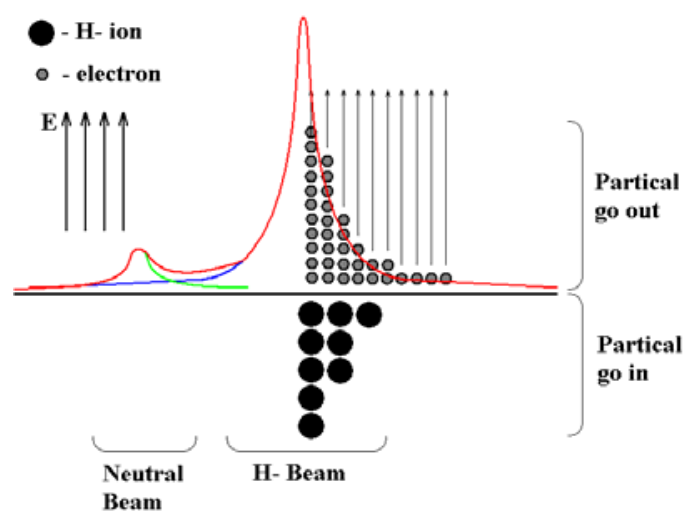

Figure 22: The model of process with positive screen voltages. 
Figures 23 and 24 show an increasing positive screen voltage and a similarly growing emittance due to more electrons being removed and accordingly a higher signal produced. Between 50 and $150 \mathrm{~V}$ the emittance is essentially flat. When the screen voltage equals $150 \mathrm{~V}$, there are no apparent shoulders or negative holes and accordingly this value for the screen voltage is optimal for collecting data.

Very interestingly, the amplitude signal is asymmetric with the emittance value (clearly in the measurements with a 25 mil probe step as in Fig. 24). This dependence means that the real amplitude is lost in measurements with very wide probe steps. In experiments with 5 mil steps this dependence is smaller. Correspondingly a smaller step size gives a better peak amplitude.

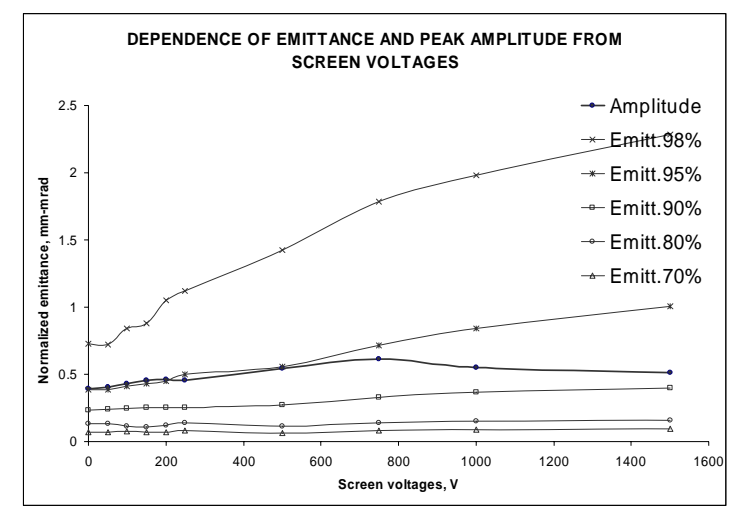

Figure 23: Dependence of emittance and peak amplitude as function of screen voltages ( 5 mil step).

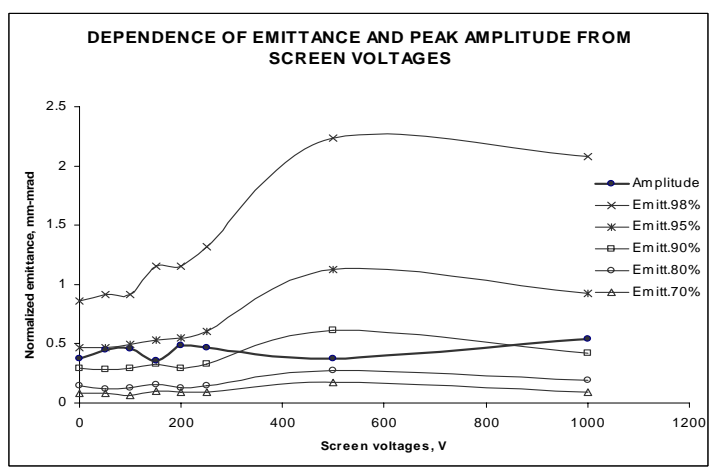

Figure 24: Dependence of emittance and peak amplitude as function of screen voltages (25 mil step).

\section{RMS EMITTANCE CALCULATION}

For RMS emittance analyses it is necessary to determine the background parameters very carefully [7].

The first task is to do a histogram analyses (Fig. 25). Current is plotted on the abscissa in arbitrary units (in this case a current is normalized to 100), on the ordinate is the number of measurements. This procedure helps to estimate the global bias for subtraction. The bias means an error in the measurement linked with different zero levels of the amplifiers and data collected with noise. The highest bias is about 0.5 , and possible bias is from 0.5 to 4 .

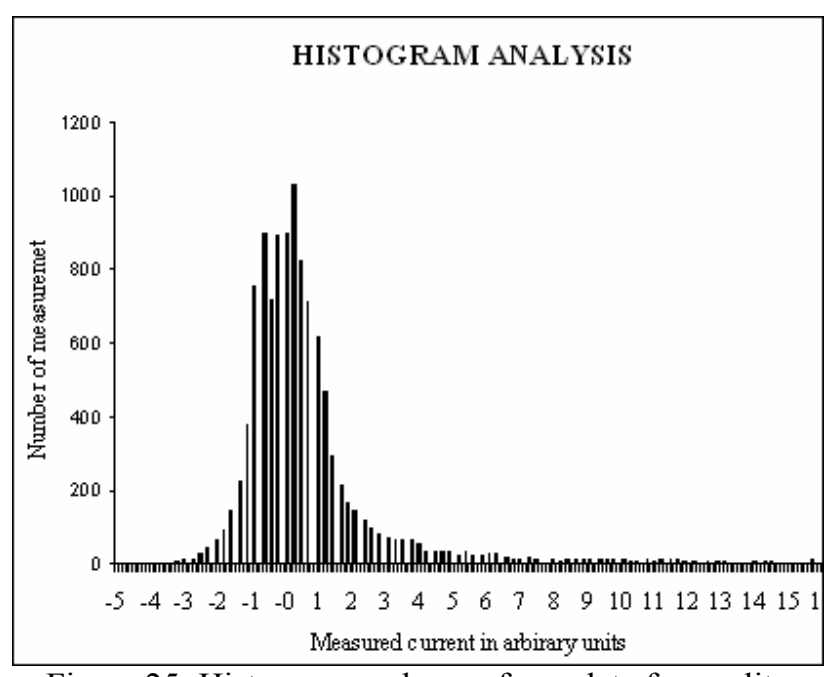

Figure 25: Histogram analyses of raw data for quality emittance estimate

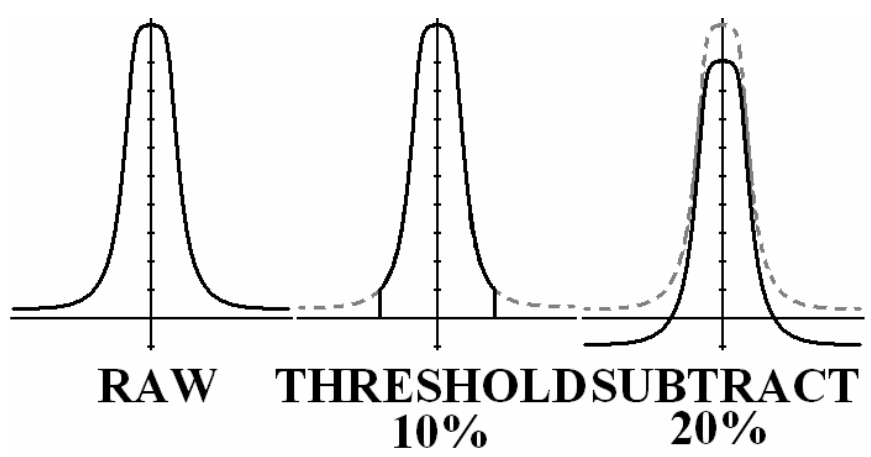

Figure 26: Steps in performing in RMS emittance

Figure 26 shows the different operations with the raw data, thresholding and subtracting bias.

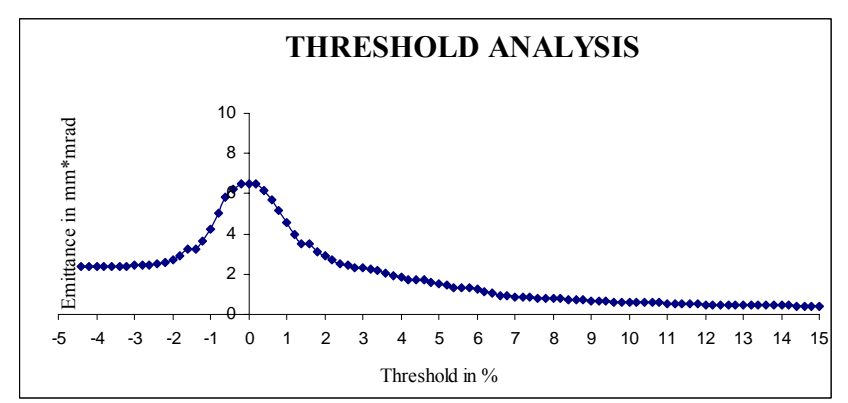

Figure 27: Threshold analysis of raw data for quality emittance estimate

The second task is to make a threshold analyses (Fig. 27). Threshold means cutting off or zeroing all data less then a desired fraction current value (threshold). This procedure can help in understanding the unbiased data. On the abscissa is the threshold in percent of the peak amplitude, on the ordinate is the rms emittance in $\pi \mathrm{mm}$ mrad. The emittance growth has a threshold from -4 to 0 $\%$, because the quantity of negative numbers decreased. After the zero value of the threshold the emittance goes 
down. This means that the obtained data is unbiased, and a zero level is correct.

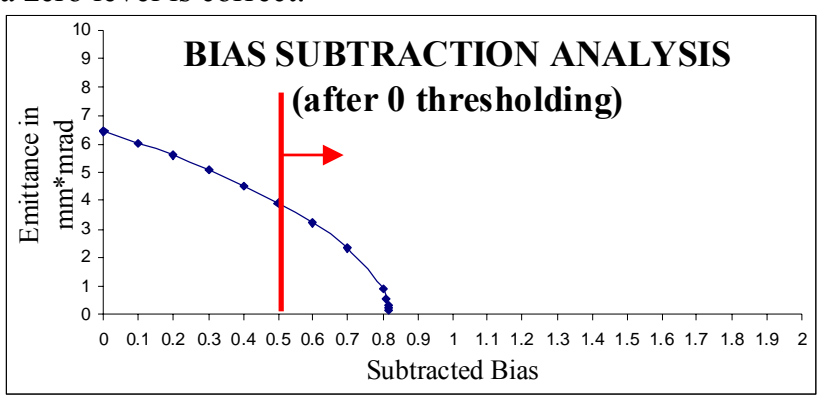

Figure 28: Bias subtraction analysis following $0 \%$ threshold of raw data

The next bias subtraction analyses (Fig. 28) shows the sensitivity of this method. After some value (in this case it is 0.82 ) the emittance will be imaginary and can not be plotted. Using the global bias estimate from the histogram analyses the emittance is less than $4.5 \pi \mathrm{mm}$-mrad. But there is some questions about the bottom bound.

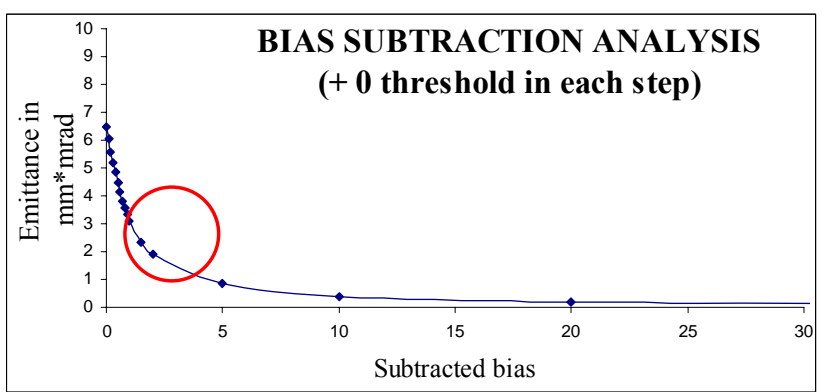

Figure 29: Bias subtraction analysis following after 0\% threshold in each step

The last analyses is a bias subtraction analyses with zero threshold in each step (Fig. 29). This type of analyses together with the histogram analyses gives a higher quality estimate. The unnormalized RMS emittance for our beam is $2.5 \pm 1.5 \pi \mathrm{mm}$-mrad.

\section{SUMMARY}

We have developed a model for ion-electron emission for a slit emittance scanner. This shows that the emittance is influenced by the screen voltage, but there is a flat region between 50 and $150 \mathrm{~V}$. The optimal screen voltage with which we collect "good" data is about 100-150V.

Also with a large step size the measurement of emittance is not proportional to the measured peak amplitude. The optimal step with which we don't lose peak data is about $5 \mathrm{mil}(1.27 \mathrm{~mm})$.

\section{REFERENCES}

[1] R. Keller "Emittance - Definitions, Procedures and Application", LANL/GSI , June 26,1985.

[2] H. Zhang "Ion sources", Science Press, Springer

[3] S. Ohnuma "The beam emittance: what we said we would have, what we think we have, and what we hope we could have", Fermilab, EXP-111, November 28, 1983

[4] K. Prelec and Th. Sluyters "Formation of Negative Hydrogen Ions in Direct Extraction Sources", Rev. Sci. Instrum., Vol 44, No10, October 1973.

[5] D. Moehs, J. Peters, and J. Sherman "Negative Hydrogen Ion Sources for Accelerators", IEEE Transaction on Plasma Science, vol. 33,no. 6, December 2005.

[6] C.Schmidt "Proton Ion Sources and Low - Energy Injectors", Cornell University PHYS 687/487: Selected Topics in Accelerator Technology, November 28, 2000.

[7]M. Stockli, R.Welton, R. Keller, A. Letchford, R.Thomae, and J. Thomason "Self-consistent, unbiased RMS-emittance estimates for data measured with a single current amplifier". 